\title{
Uni- and Biaxial Impact Behavior of Double-Gated Nanoclay-Reinforced Polypropylene Injection Moldings
}

\author{
Valeria Pettarin, ${ }^{1}$ Gastón Viau, ${ }^{1}$ Laura Fasce, ${ }^{1}$ Julio C. Viana, ${ }^{2}$ Antonio J. Pontes, ${ }^{2}$ \\ Patricia M. Frontini, ${ }^{1}$ Antonio S. Pouzada ${ }^{2}$ \\ ${ }_{1}^{1}$ Polymer Engineering \& Science Group, Institute of Materials Science and Technology (INTEMA), \\ CONICET-University of Mar del Plata, Argentina
}

${ }^{2}$ Institute for Polymers and Composites/I3N, University of Minho, Guimarães, Portugal

\begin{abstract}
Polypopylene/nanoclay three-dimensional parts were produced without intermediate steps by direct injection molding to explore the influence of flow features and nanoclay incorporation in their impact performance. The nanocomposite was obtained by direct compounding of commercial PP with nanoclay masterbatch. The as-molded morphology was analyzed by X-ray and TEM analyses in terms of skin-core structure and nanoclay particle dispersion. The nanoclay particles induced the reduction of $\beta$-form spherulites, a known toughener. The impact behavior was assessed in tensile and biaxial modes. The PP nanocomposite molding toughness was practically unaffected by the processing melt temperature and flow rate. Conversely the nanoclay presence is influent in the impact performance. Under biaxial stress impact, the regions close to weld lines are tougher than the bulk and the fracture develops with main crack paths along the flow direction and the weld line. Cracking along the weld line results from less macromolecular interpenetration and chain entanglement, and unfavorable nanoparticle orientation. It seems that a failure mechanism which involves nanoclay delamination and multiple matrix crazing explains the toughening of PP in the directions where the nanoparticle orientation with respect to loading is adequate. POLYM. ENG. SCI., 53:724-733, 2013. (C) 2012 Society of Plastics Engineers
\end{abstract}

\section{INTRODUCTION}

Polypropylene (PP) is one of the more used commodity thermoplastics. Great efforts are undertaken to transform it into a material suitable for high performance engineer-

Correspondence to: Patricia M. Frontini; e-mail: pmfronti@fi.mdp.edu.ar or Antonio S. Pouzada; e-mail: asp@dep.uminho.pt

Contract grant sponsors: CONICET, ANPCyT from Argentina, MINCyT (Argentina) - FCT (Portugal), Universities Nacional de Mar del Plata and Minho.

DOI 10.1002/pen.23306

Published online in Wiley Online Library (wileyonlinelibrary.com). (C) 2012 Society of Plastics Engineers ing applications. Research work has therefore been focused on upgrading the mechanical performance of PP by toughening (to overcome the moderate low temperature impact resistance) by filling and reinforcing with a second phase (to increase stiffness, strength and temperature resistance, and to decrease shrinkage) or both [1].

Polymeric nanocomposites emerged as new materials showing potential for industrial applications and quite exceptional properties at low filler contents [2, 3]. Layered silicate polymer nanocomposites are claimed to have multiple advantages. An important commodity polymer such as PP, when lightly filled with nanoclay (typically less than $5 \mathrm{wt} \%$ ), can be used in applications with requirements typical of engineering plastics with additional advantages (e.g., lower density). However, only well-dispersed and well-exfoliated nanoparticles can lead to the expected improvement of properties [4-6]. Therefore raw material producers, converters, and end-users have to tackle both compounding and processing issues. Surface modification of nanofillers with organic surfactant and adaptation of compounding conditions (high shear, high residence time, special screw profile design in case of melt compounding for example) may help to get rid of most of compounding issues.

However, to make nanocomposites economically viable, the parts must be produced in common processing equipment eliminating additional steps [7]. Following this idea, novel masterbatches (MB) have been developed. MB producers claim that they include all compatibilizers needed to promote complete nanoclay dispersion, easy processability and compatibility with standard equipment, including extruders, mixers, and injection molders. The final injection-or extrusion-molded part should be easily obtained by mixing/diluting the MB in the appropriate polymeric matrix.

Injection molding is the soundest process to manufacture plastic parts, since complex geometries become available in one automated production step. The influence of processing parameters is critical in the performance of 
these products (e.g. [8-10]). Injection molding induces high molecular orientation which can affect part performance. Moreover, if weld lines, an unavoidable reality in the injection molding of complex parts, are likely to occur, things become more complicated. Multiple gating, splitting of the melt flow due to inserts in the impression or through holes, as well as changes of thickness give rise to regions in the impression where the melt flow fronts recombine and weld. This imperfection is not only aesthetically unattractive, but it is also in the weld line region where the properties are different from the bulk [9, 11]. There exists a large amount of documentation of studies on the mechanical characterization of molded items at the weld line and on the weld line morphology, especially in fiber reinforced polymers [12]. However, despite the continuous usage of particulates as polymer reinforcement, characterization of the weld line in particle-reinforced composites has not been as extensive. For instance, it is known that the alignment of fibers along the weld line in fiber reinforced composites is the key feature inhibiting the reintegration of multiple flow streams, thus resulting in poor bonding of the colliding melt interfaces. It is also known that the stagnation flow which results when the melt fronts impinge upon one another causes the weld line to behave as a solid wall on which fibers are continuously deposited throughout the filling process [13]. Nevertheless, it is not clear how these mechanisms operate in the case of particulate composites, even less in the case of layered silicate composites up to the authors' knowledge.

Impact toughness is often one of the fundamental material requirements used to measure the performance of plastics products. Energy absorption is an increasingly important function of structural materials for several reasons. For example, structural crashworthiness is now an essential requirement in the design of automotive vehicles. The crashworthy structure is designed such that in the event of a crash, it absorbs the impact energy in a controlled manner before the energy gets transmitted into the occupant. The use of PP nanocomposites in interiors, such as pillars, door trims, and dashboards, should lead in this direction. As polymer-based nanocomposites are envisaged to have improved toughness, it is important to determine performance of final parts under impact conditions.

This work explores the possibility of producing high performance $\mathrm{PP} /$ nanoclay parts by direct injection molding without intermediate steps, and analyses the effect of complex flow inside the mold, i.e. the effect of injection points and weld line formation. To this aim injection molded PP/nanoclay parts were produced by direct compounding of commercial PP and a nanoclay-based masterbatch (MB). Microstructure was studied by transmission optical microscopy (TOM), transmission electronic microscopy (TEM), X-ray diffraction (XRD), and depthsensing indentation. Uniaxial and biaxial impact behavior at different locations of injection molded parts was assessed and analyzed.

\section{EXPERIMENTAL}

\section{Materials}

This study was carried out on a propylene homopolymer, F-045 D2 (from Sunoco Chemicals) with MFI of 4.9 $\mathrm{g} / 600 \mathrm{~s}$ at $230^{\circ} \mathrm{C} / 2.16 \mathrm{~kg}$, and a commercial MB of PP with $50 \%$ of organoclay, Nanomax-PP P-802 (from Nanomax Polyolefin Masterbatch Products).

\section{Injection Molding Program}

Nanocomposites were obtained by direct injection of mixtures of PP and nanoclay rich MB. Different amounts of incorporation of nanoclays were used by diluting the $\mathrm{MB}$ in the PP matrix. Rectangular boxes of $1.4 \mathrm{~mm}$ thickness were injection molded in a double-gated hot runner injection mold using a Klöckner Ferromatic FM20 injection machine of $200 \mathrm{kN}$ clamping force. Hot runner molds are adequate to mass production as in the automotive industry. These molds lead to the reduction of production costs by saving material of the nonrequired gating system and by shortening the cycle time. Furthermore lower injection pressure is required, the process temperature is controllable with greater precision, more uniform filling is achievable in multi-impression molds, and thus, the final mechanical properties of the moldings are improved [14]. In the mold used, the two gates that are equidistant from the mold axis generate a weld line in the centre of the molding, allowing studying also the effect of weld lines on part performance. The injection pressure was monitored during injection by a sensor in the mold cavity. The processing conditions are listed in Table 1.

A design of experiments based on the Taguchi approach [15] was applied to determine the most influential molding parameters that will yield better impact response of the injected boxes. Three molding parameters were selected: masterbatch content $(\mathrm{MB})$, melt temperature $\left(T_{\mathrm{m}}\right)$, and injection rate $\left(Q_{\mathrm{j}}\right)$. As the influence of these factors may vary non-linearly, each factor was assigned with three levels. According to the level of each parameter, an L9-orthogonal table was used to establish the molding program (Table 2). The nine conditions were repeated with unfilled PP moldings for comparison. Additional pieces were injected according to experiment \#2 with various MB nominal contents of $2,6,10$, and $14 \mathrm{wt} \%$.

TABLE 1. Processing settings.

\begin{tabular}{lc}
\hline \multicolumn{1}{c}{ Processing parameter } & Value \\
\hline Injection temperature $\left({ }^{\circ} \mathrm{C}\right)$ & 235 \\
Screw rotation velocity $(\mathrm{rpm})$ & 250 \\
Injection rate $\left(\mathrm{cm}^{3} / \mathrm{s}\right)$ & 25.8 \\
Hydraulic packing pressure $(\mathrm{kPa}) / \mathrm{time}(\mathrm{s})$ & $300 / 10$ \\
Cooling time $(\mathrm{s})$ & 15 \\
\hline
\end{tabular}


TABLE 2. Design of experiment and factors and levels for the injection molding experiments.

\begin{tabular}{lcrr}
\hline $\begin{array}{c}\text { Experiment } \\
(\#)\end{array}$ & $\begin{array}{c}\text { Nominal MB content/ } \\
\text { nominal nanoclay content }(\%)\end{array}$ & $T_{\mathrm{m}}\left({ }^{\circ} \mathrm{C}\right)$ & $Q_{\mathrm{j}}\left(\mathrm{cm}^{3} / \mathrm{s}\right)$ \\
\hline 1 & $6 / 3$ & 205 & 25.8 \\
2 & & 220 & 90.3 \\
3 & $10 / 5$ & 235 & 103.2 \\
4 & & 220 & 103.2 \\
5 & & 235 & 25.8 \\
6 & $14 / 7$ & 205 & 90.3 \\
7 & & 235 & 90.3 \\
8 & & 205 & 103.2 \\
9 & & 220 & 25.8 \\
\hline
\end{tabular}

\section{Molding Microstructure}

Polarized light microscopy (PLM) was used to observe the microstructure of the moldings. $15 \mu \mathrm{m}$ thick samples were cut with a Leitz 1401 microtome and observed with an Olympus $\mathrm{BH} 2$ polarized light microscope.

Distribution and exfoliation level of clay were evaluated by TEM and XRD. TEM microphotographs were obtained with a Jeol 100 CX microscope using acceleration voltage of $200 \mathrm{kV}$. Samples were ultramicrotomed at room temperature with a diamond knife to a $70 \mathrm{~nm}$ thick section. XRD analysis was performed on samples from the skin layer using a Phillips X'PERT MPD diffractometer $(\mathrm{CuK} \alpha$ radiation $\lambda=1.5418 \AA$, generator voltage $=40$ $\mathrm{kV}$, current $=40 \mathrm{~mA}$ ). Measurements were recorded every $0.02^{\circ} \theta$ steps for $1 \mathrm{~s}$, each varying $2 \theta$ from 2 to $40^{\circ}$. The interlayer distance of the nanoclay was calculated from the (001) peak using the Bragg's law $\left(\lambda=2 d_{001} \sin \theta_{001}\right)$.

From the wide angle X-ray diffraction (WAXD) pattern, the PP $\alpha$-phase orientation indices were determined as [16]:

$$
\begin{gathered}
A_{110}=\frac{I_{\alpha_{1}}}{I_{\alpha_{1}}+I_{\alpha_{4}}} \\
A_{040}=\frac{I_{\alpha_{2}}}{I_{\alpha_{1}}+I_{\alpha_{2}}+I_{\alpha_{3}}}
\end{gathered}
$$

where I is the peak height after background subtraction, $\alpha_{1}$ corresponds to the (110) reflection, $\alpha_{2}$ to the (040) reflection, $\alpha_{3}$ to (130) reflection and $\alpha_{4}$ to the (111) and (041) reflections. The $A_{110}$ index is based on the extinction of (hk1) reflections (in which case, $A_{110}$ tends to (1) relatively to the (110) reflections. It thus gives an indication of an orientation roughly parallel to the c-axis of the crystallites corresponding to the flow direction in the injected samples [17]. The $\mathrm{A}_{040}$ index is based on the extinction of the (hk0) reflections relatively to the $(0 \mathrm{k} 0)$ reflections and gives an indication of a preferential orientation perpendicular to the $b$-axis, i.e. pretty much parallel to the surface sample [17].

Depth sensing indentation measurements were performed using a Triboindenter Hysitron equipped with MRNP device to determine the distribution of surface mechanical properties through the sample thickness in PP and nanocomposites injection moldings. Samples were cut from the pieces, included in an epoxy resin block and polished with increasing grades of silicon carbide paper. Indentations were made using a diamond Berkovich tip under load controlled conditions. A trapezoidal loading function was used to minimize creep effects on the unloading response. A maximum load of $20 \mathrm{mN}$ was applied at a rate of $2 \mathrm{mN} / \mathrm{s}$, then the load was held during $20 \mathrm{~s}$ and finally the load was removed at $2 \mathrm{mN} / \mathrm{s}$. A pattern of 25 indentations was applied to cover all the piece thickness and repeated at four different locations on each sample.

Indentation load-penetration depth data were analyzed following the procedure outlined by Oliver and Pharr [18] to obtain the reduced elastic modulus, $E_{\mathrm{r}}$, and the indentation hardness, $H$.

\section{Impact Tests}

The impact properties of the moldings at room temperature were assessed by means of uniaxial tensile (inplane) and biaxial flexural (out-of-plane) tests.

The effect of the weld lines on the mechanical properties of a given polymer is usually estimated by testing in impact mode tensile specimens molded with and without weld line. To study this effect, tensile-impact tests were carried out at room temperature using a standard pendulum Ceast 6545 with energy of $7.5 \mathrm{~J}$ and fitted with ancillaries for tensile-impact testing, at room temperature. The mpact velocity was of $3.6 \mathrm{~m} / \mathrm{s}$ according to ISO 8256 . Rectangular samples $(50 \mathrm{~mm} \times 100 \mathrm{~mm})$ were cut with a G-Weike LG900 laser engraver at different locations in the moldings, as shown in Fig. 1a. Three samples were cut at different positions along the region of weld line formation. In these positions the fluxes moving from the

(a)
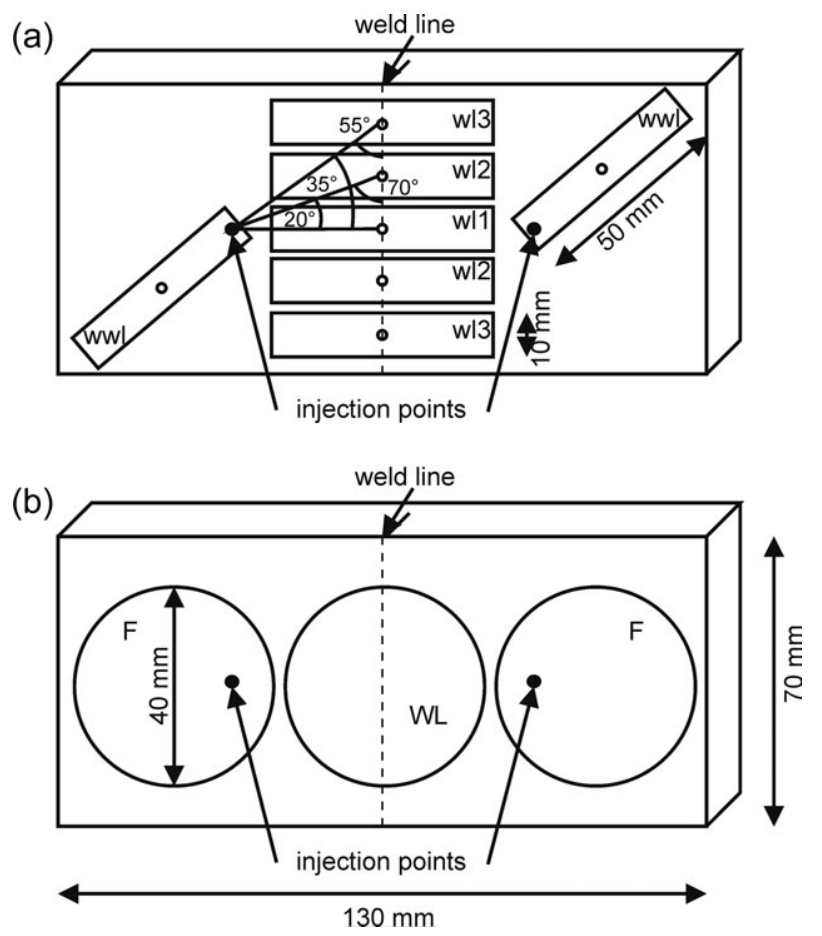

FIG. 1. Location of (a) tensile and (b) disc test specimens. 
injection points

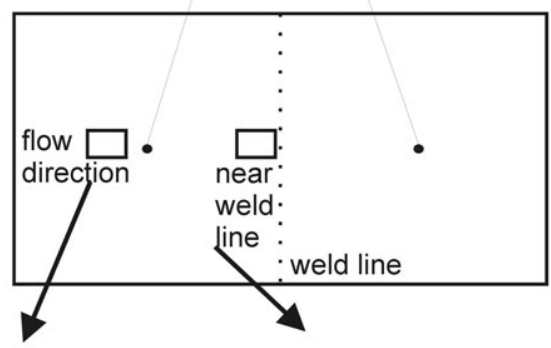

(a)

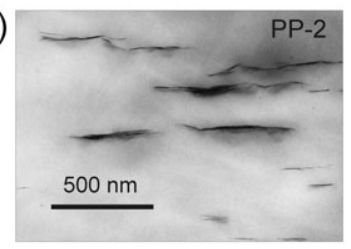

(b)

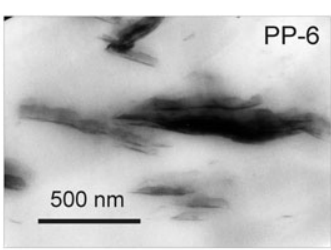

(c)

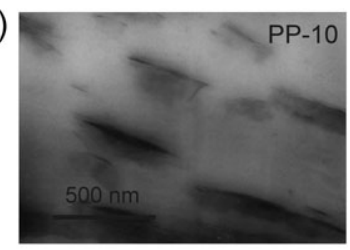

(d)

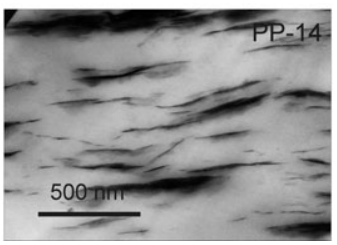

(e)

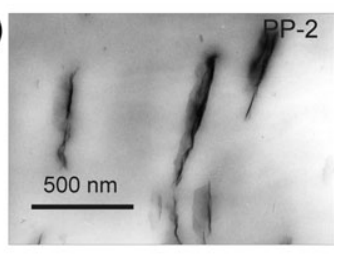

(f)

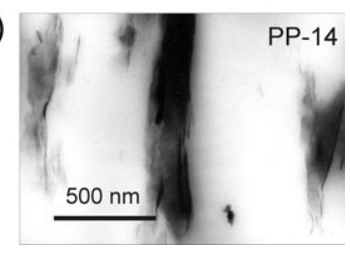

FIG. 2. Nanoclay dispersion in PP nanocomposites. TEM pictures obtained in the flow direction for (a) PP-2, (b) PP-6, (c) PP-10, and (d) PP-14; and near the weld line zone for (e) PP-2 and (f) PP-14.

injection points collide at different angles: WL1 $180^{\circ}$, WL2 $140^{\circ}$, and WL3 $110^{\circ}$. One sample was cut away from the weld line, in a radial position along the flow direction (WWL). A $2 \mathrm{~mm}$ hole was drilled at the centre of the samples to induce stress concentration and the breaking of specimens in the same zone. The tensile impact toughness was calculated as the energy to break the specimen divided the ligament area.

Biaxial impact tests are tests on representative samples, rather than measurements of basic material properties on standard test pieces, which give a realistic view of inservice impact situations-being closer to real life conditions-with the additional advantage that they provide a convenient method of studying changes induced by flow in molded part performance [19-21]. Biaxial impact tests (with loading perpendicular to the plane containing one of the injection molding points) were carried out according to ASTM D 3763-93 in two locations of the pieces (on (WL)

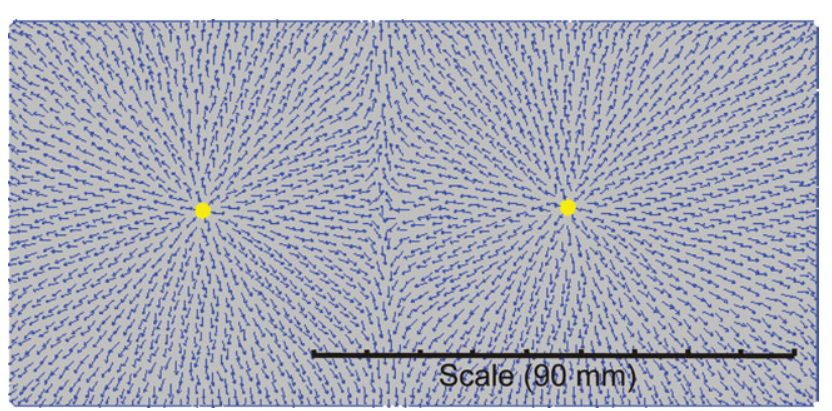

FIG. 3. Flow direction field (normal to the advanced flow front) as simulated by Moldflow. [Color figure can be viewed in the online issue, which is available at wileyonlinelibrary.com.]

and out (F) of weld line) (Fig. 1b) using an instrumented Ceast Fractovis 6787 falling weight equipment. The specimens were clamped between two steel plates with a circular opening of $40 \mathrm{~mm}$ in diameter and tested at $1 \mathrm{~m} \mathrm{~s}^{-1}$ and room temperature. The biaxial impact toughness, $J$, was calculated as the total energy to break the sample (total area over the force-displacement curve) divided thickness.

\section{RESULTS AND DISCUSSION}

\section{Microstructure of Moldings}

The dispersion of clay in PP nanocomposites systems is visible in TEM images (Fig. 2). It is seen that the dispersion of clay is uniform and there is apparently no indication of aggregation of clay (big tactoids). Clay platelets are oriented in the flow direction inside the impression, this direction being assessed by mold filling simulation using the software Autodesk Moldflow (Fig. 3). XRD patterns illustrate the intercalation of polymer between the galleries of clay layers (amplification of low $2 \theta$ as shown in Fig. 4). The intergallery space is $13.4 \mathrm{~nm}$ for all systems.

XRD results indicate that the presence of $\mathrm{MB}$ induced changes in the crystal structure of PP (Fig. 4). In PP moldings a small amount of $\beta$-form (peaks at $2 \theta=16.2$ and $21.2^{\circ}$, associated to (300) and (301) planes) was found, besides the main $\alpha$-form crystallites (peaks at $2 \theta=$ $14.2,17,18.8$ y $25.5^{\circ}$, corresponding to (110), (040), (130), and (131) planes, respectively) [22]. In the nanocomposite moldings, the intensities of peaks at $16.2^{\circ}$ and $21.2^{\circ}$ ( $\beta$ polymorph) were attenuated, while the intensity peaks at $17^{\circ}$ and $25.5^{\circ}$ ( $\alpha$ polymorph) were enhanced. It seems therefore that $\mathrm{MB}$ acted as $\alpha$ nucleation site, reducing the amount of $\beta$-form PP. Similar results of $\beta$-suppressor effect of filler were reported by Varga et al for PP-talc composites [23]. Another important feature is the increase of the $(040) /(110)$ ratio, which indicates that MB induced the orientation of the crystalline-phase of PP in the (040) direction [24]. Orientation indices based on the reflection intensities were determined and the values are plotted in Fig. 5. The results confirm that the presence of nanoclay caused the orientation of $\alpha$-PP crystals in the (040) direction which means preferential orientation per- 


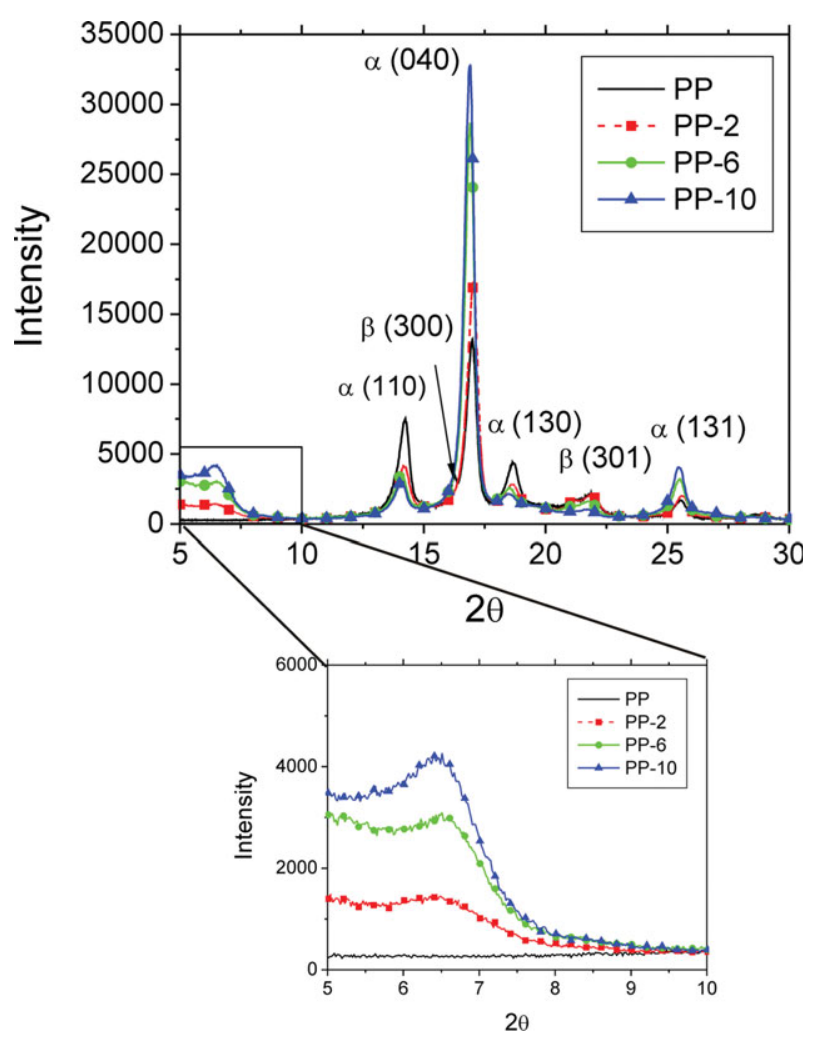

FIG. 4. XRD patterns of PP nanocomposites injected as for experiment $\# 2$. [Color figure can be viewed in the online issue, which is available at wileyonlinelibrary.com.]

pendicular to the b-axis of the crystallites, i.e. parallel to the surface sample.

The skin-core structure of the moldings was revealed by PLM (Fig. 6) and also by depth sensing indentation data (Fig. 7). Near the cold mold surface a frozen layer is formed adjacent to a shish-kebab or row structure developed, probably, in the region of highest shear rate. The

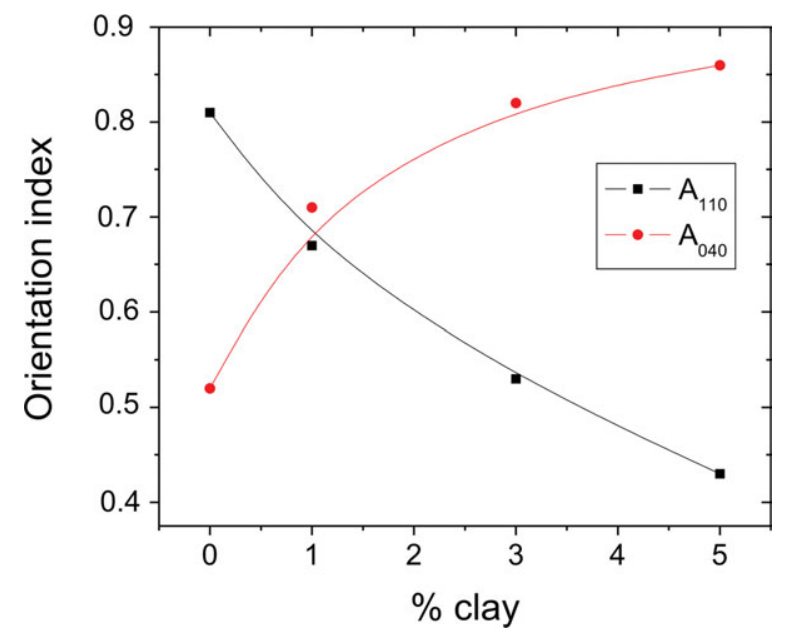

FIG. 5. Orientation indices of $\alpha$-PP from XRD patterns. [Color figure can be viewed in the online issue, which is available at wileyonlinelibrary. com.] (a)

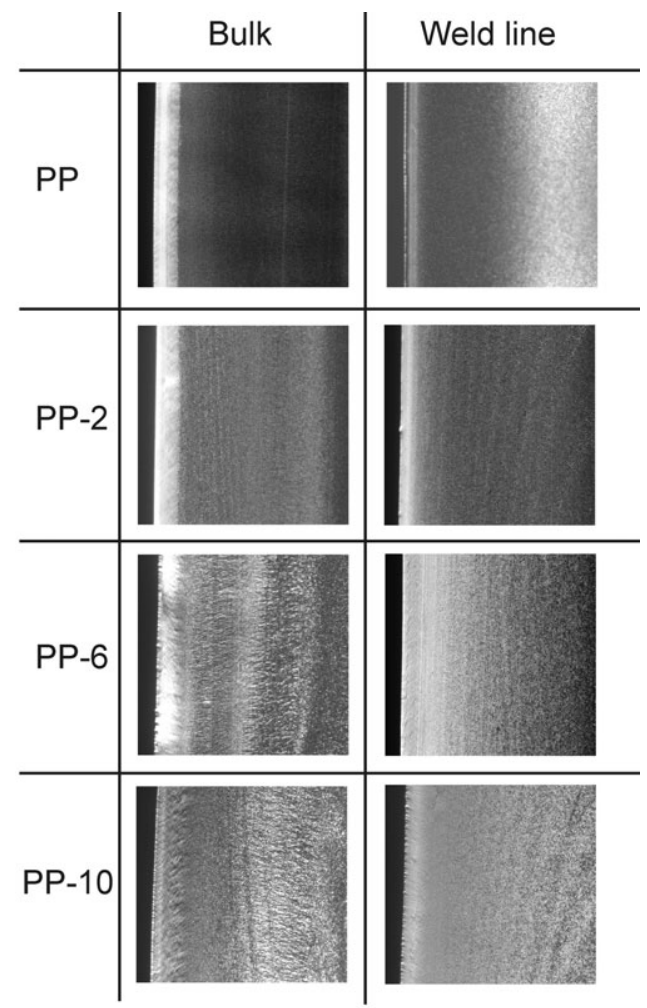

(b)

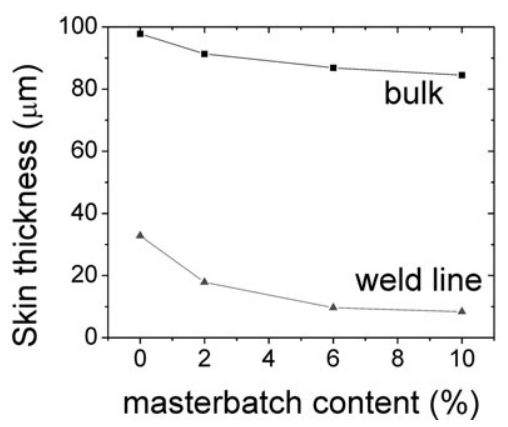

FIG. 6. (a) Microstructure of PP nanocomposites moldings as experiment \#2 observed by polarized light microscopy. (b) Variation of skin thickness with incorporation of MB.

shish-kebab structure is common in PP and other polyolefins and is a consequence of the fast crystallization of the polymer chains under high stresses near the skin region, as described by Fujiyama et al. [25]. In the vicinity of this sheared layer, $\beta$-spherulites can be found as a result of the crystallization under shear. In the core, $\alpha$-spherulites are formed under quiescent crystallization conditions. A decrease of the skin thickness with the increase in MB content and the distance from the injection point was observed also by PLM (Fig. 6b). The latter is in accord with Fujiyama's work that stated that the crystalline structure and thickness of the skin change along the dimensions of the molded plaque [26-28]. The surprising finding is that the skin thickness decreases with MB content. It seems that the nanoclays reduce PP macromolecules relaxation time and therefore skin thickness. Also shear dissipation is higher further reducing the skin thickness. 
(a)

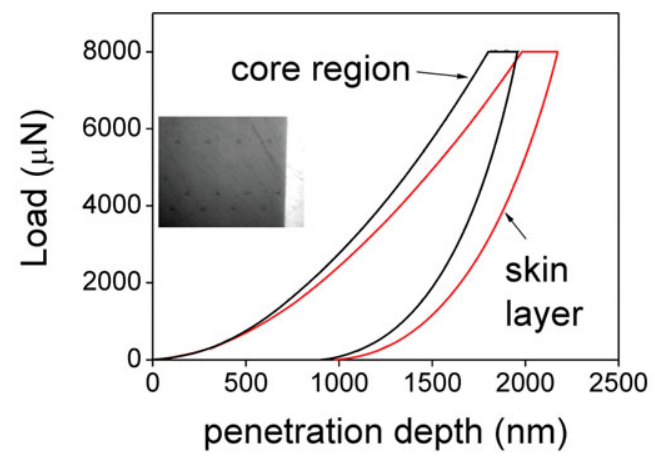

(b)
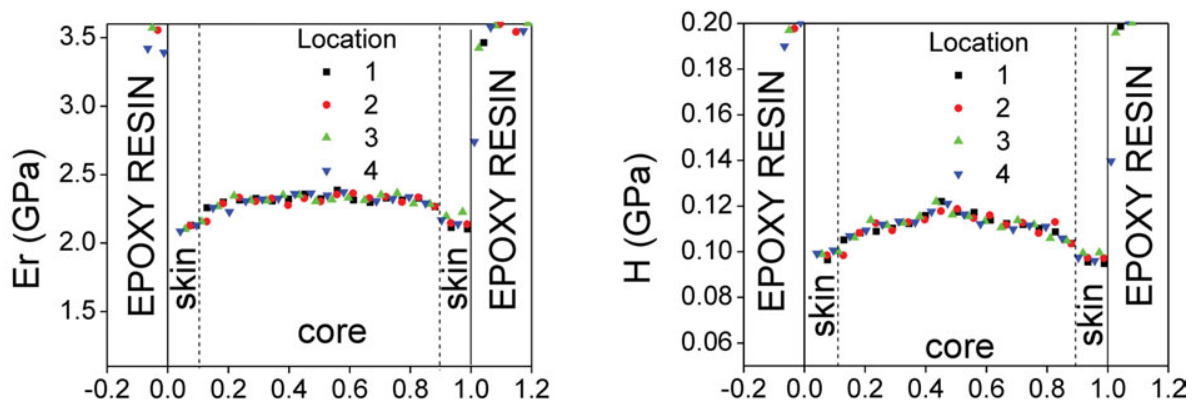

Relative thickness position

Relative thickness position

(c)

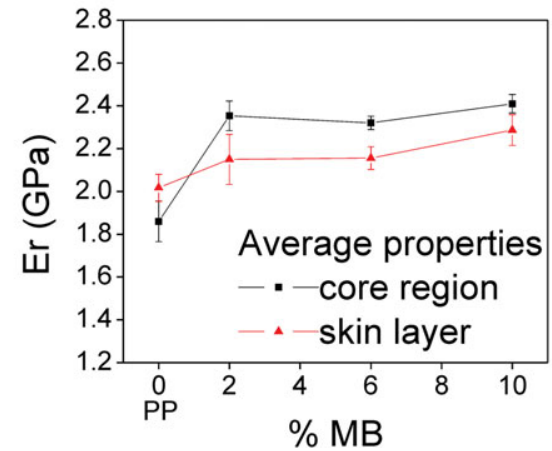

(d)

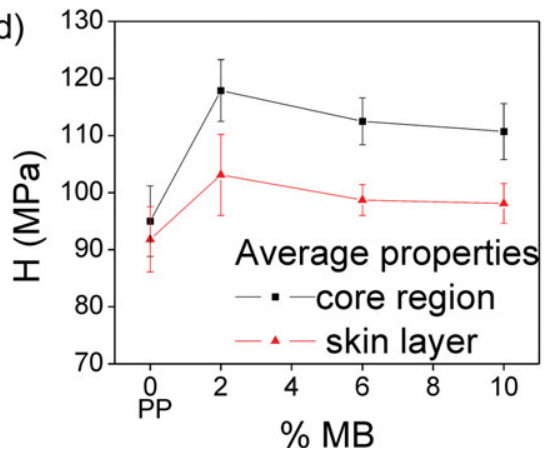

FIG. 7. Depth sensing indentation results. (a) Typical indentation load-penetration depth curves for PP-6 sample in the skin and core. (b) Er and H profiles through the thickness for PP-6 sample. (c) Average values of Er as a function of $\mathrm{MB}$ content in the skin and core. (d) Average values of $\mathrm{H}$ as a function of $\mathrm{MB}$ content in the skin and core. [Color figure can be viewed in the online issue, which is available at wileyonlinelibrary.com.]

The indentation data through the thickness do confirm the presence of the skin layer in all nanocomposites (as shown in the example of Fig. $7 \mathrm{a}$ and $\mathrm{b}$ ). The core region is stiffer and harder than the skin region (Fig. 7c and d). This difference is due to the higher crystallinity of the core in comparison with the skin. The addition of $2 \% \mathrm{MB}$ caused an increase in the elastic modulus and hardness, due to the $\alpha$-nucleating effect of the nanoclay and the differences in crystalline structure induced by the presence of MB. Further incorporation of MB did not affect the stiffness, probably due to the compensation effect between clay and compatibilizer (PP-MAN) present in MB. MB content larger than $2 \%$ induced only a slight decrease in hardness, indicating a slight reduction in yield stress. The nanoclay stacks provide microvoid nucleating sites at their surfaces and therefore release the constraints for shear yielding [29].

\section{Influence of Processing Conditions on Impact Toughness}

The effect of processing factors on the tensile and biaxial impact toughness was ascertained following an ANOVA analysis as suggested by Keppel and Zedeck [30] and is illustrated in Fig. 8. The results show that the MB content is the most influencing parameter, and the tensile-impact toughness is practically unaffected by the selected processing conditions. In this case, the total energy absorbed during impact away from the weld line is higher, this feature being a weak point on the molding. The weld line location \#3 is always the weakest point. This zone is the most distant from the gates, and corresponds to the points where the temperature and pressure at the joining flow fronts are lowest, as confirmed by Moldflow analysis. Interestingly, the biaxial impact toughness in the weld line is higher than out of weld line zone. 
(a)

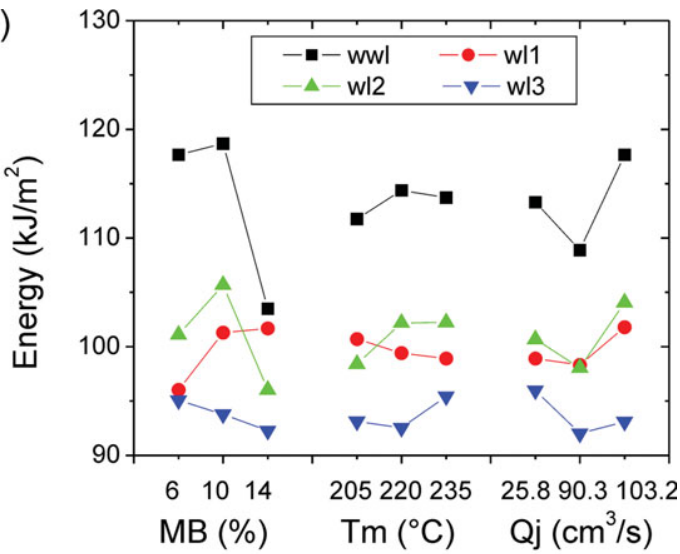

(b)

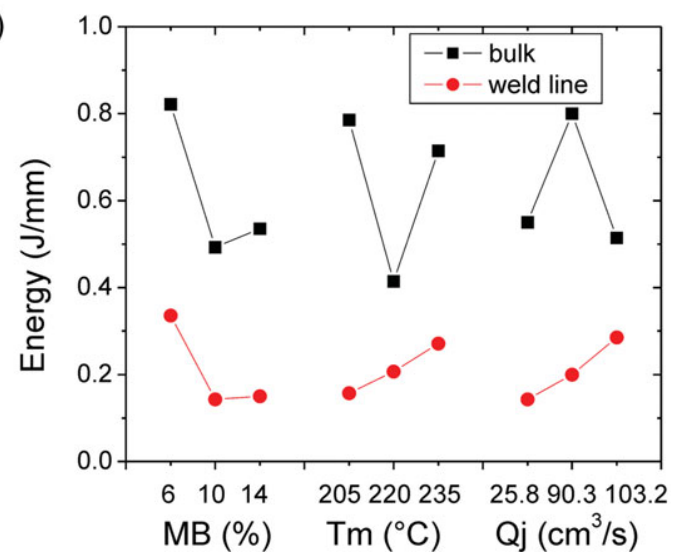

FIG. 8. Effects of processing parameters on impact toughness under (a) tensile impact and (b) biaxial impact. [Color figure can be viewed in the online issue, which is available at wileyonlinelibrary.com.]

Pressure data inside the mold impression are shown in Fig. 9 for various percentages of MB. The addition of MB increases the melt viscosity which causes the maximum pressure inside the mold being lower. From these curves it may also be deduced that the part freezes sooner with the incorporation of $\mathrm{MB}$, and this is dependent on the amount of MB. Adding the nanoclay may increase the thermal diffusivity of the material and also the viscosity, thus reducing the cooling time. Differences in pressure curves due to different MB content are not significant, indicating that the viscosity variation with the percentage of nanoclay in this range should be very small. This suggests that the variations in mechanical properties are mainly due to the MB content effect upon the molding morphology developed upon cooling.

\section{Influence of the Weld Line on Impact Toughness}

The impact toughness under tensile and biaxial impact conditions as a function of position in box and clay content are depicted in Fig. 10.

Under tensile conditions, the amount of absorbed energy is lower at the weld line than away from it and in the flow direction, as expected (Fig. 10a). It is possible to verify that the impact toughness tends to increase from a minimum associated to the sample WL1, where there is a frontal hit of the flow fronts, to WWL, the reference sample in a location well away from the weld line in the flow direction. This trend results from the filling process of the molding. It is useful to recall here the velocity profiles in the moldings, as depicted in Fig. 3, and the position of the weld line with respect to the loading direction. It can be observed that the angle of the weld line plane with respect to flux direction varies from a maximum of $90^{\circ}$ (sample WL1 with frontal hit of flow fronts) to a minimum of $55^{\circ}$ (sample WL3). High aspect ratio particles were shown by other authors $[31,32]$ to orientate in the plane of the weld line and parallel to the weld line, this being also supported by our TEM observations (Fig. 2). Thus, in the sample WL1, the polymer chains and the nanoclay platelets are oriented in a plane transversal to the applied force, thus contributing to the weakness of the weld line. In the other samples (WL2 and WL3), the weld line is not exactly perpendicular to the imposed tensile loading, which attenuates the depreciative effect of the weld line. Furthermore, the weld line WL1 is formed at higher melt temperature and holding pressure, this being a further contribution for a better impact performance. The samples WWL show the best performance because of the absence of the weld line and the loading being applied in the flow direction (and, expectantly, along the highest molecular orientation). In general the incorporation of MB is beneficial for the impact toughness with weld lines. The only exception is the sample WL3, where the addition of MB is detrimental. Percentages of nanoclay between 3-5 wt $\%$ lead to the best results.

On the contrary, under biaxial conditions the weld line zone of PP moldings exhibited better impact toughness than nearby the injection point (Fig. 10b). Force-displacement curves show also a more ductile behavior at the

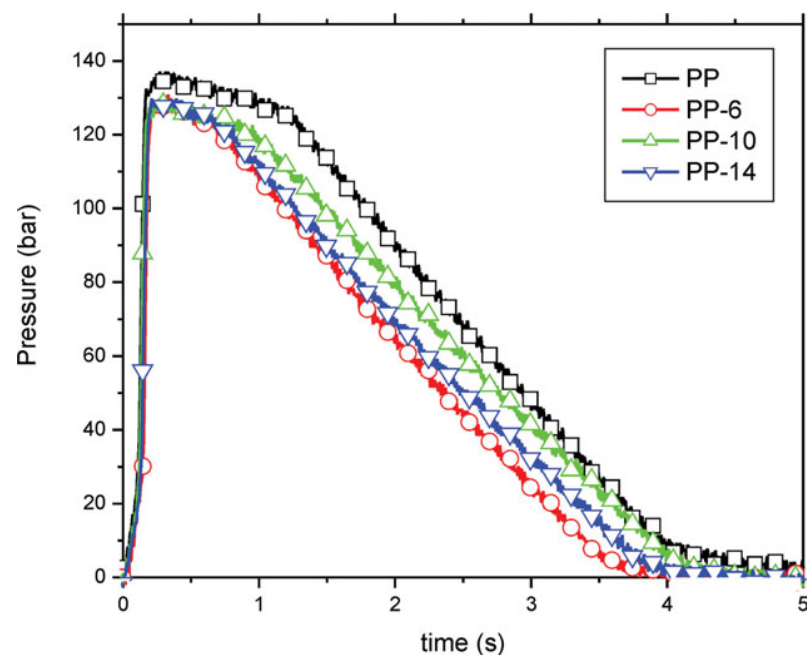

FIG. 9. Effects of nanoclay content on pressure developed inside the impression. [Color figure can be viewed in the online issue, which is available at wileyonlinelibrary.com.] 

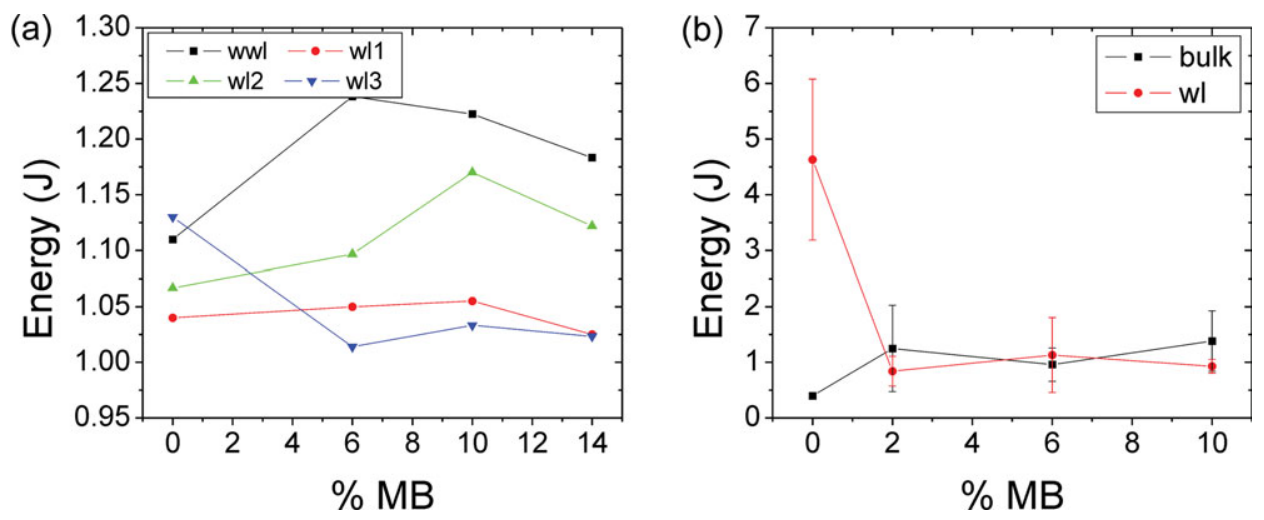

FIG. 10. Impact toughness as a function of position in box and nanoclay content. (a) Tensile loading; (b) biaxial loading. [Color figure can be viewed in the online issue, which is available at wileyonlinelibrary. com.]

weld line (Fig. 11) (similar findings were reported by Bucknall [33]). This test does not impose a preferential direction of failure of the specimen and isotropic or orthotropic materials are expected to display a symmetric deformation pattern with respect to the impact site. The failures are originated at the weakest point in the sample and propagate from there due to the high radial and circumferential tensile stresses [34]. The macroscopic analysis of the fractured surfaces shows that the fracture patterns at the weld line and close to the injection points are significantly different (Fig. 12). In samples impacted near the injection point of unfilled PP moldings, bending along the clamping ring is obvious, and the plate failed by single splitting along the melt flow direction. Conversely, in samples fractured at the weld line zone some cracks are seen running radially from the central point of impact, while others follow a circular path around the same point (circular cracks occur where the deflection caused by the striker produces a large bending moment), and there is evidence of plastic deformation shown by the stress whitening. It can be therefore concluded that the molecular orientation induced by melt flow prevailed, and the weld line contribution was not the determinant of PP molding toughness. Regarding PP/nanoclay moldings, it was found that samples exhibited similar toughness near the injection point and at the weld lines (Fig. 10b). It is clearly seen that there is not only a main crack path along the melt flow direction but also a main crack path along the weld line (Fig. 12). This result is a consequence of the lower flow interpenetration and chain entanglement at the weld line, because of the viscosity increment resulting from the nanoclay addition, and unfavorable orientation of those particles in the weld line plane. However, there is not a depreciation of the impact toughness due to weld line formation. It seems that in PP nanocomposites the toughness is determined by the molecular and particle orientations induced by flow, and the weld line does not have a determinant effect.

\section{Influence of Clay Content on the Impact Toughness}

In biaxial tests, the difference in impact toughness with nanoclay content is negligible away from the weld line. In uniaxial tests and away from the weld line, impact toughness increases with MB content, but the toughening effect of MB depends on the test direction. It is evident that toughening effect of MB on PP is not isotropic, and it depends on nanoclay orientation.

The nanoclay toughening of polymers has been attributed to the local and global conformation of the polymers within the host galleries of nanoparticles being signifi-
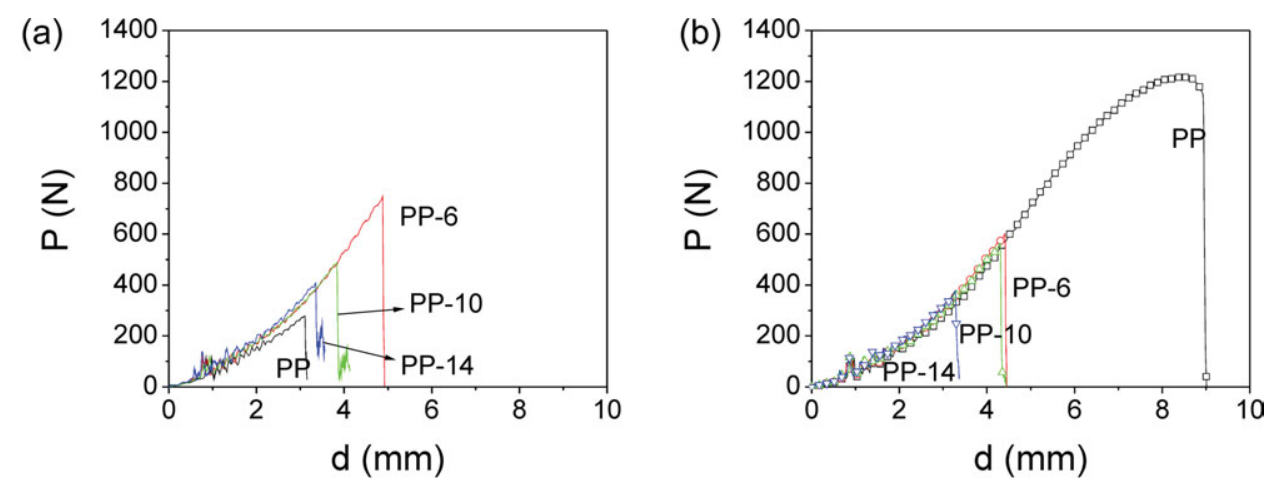

FIG. 11. Force-deflection curves under biaxial impact in: (a) bulk; (b) weld line. [Color figure can be viewed in the online issue, which is available at wileyonlinelibrary.com.] 


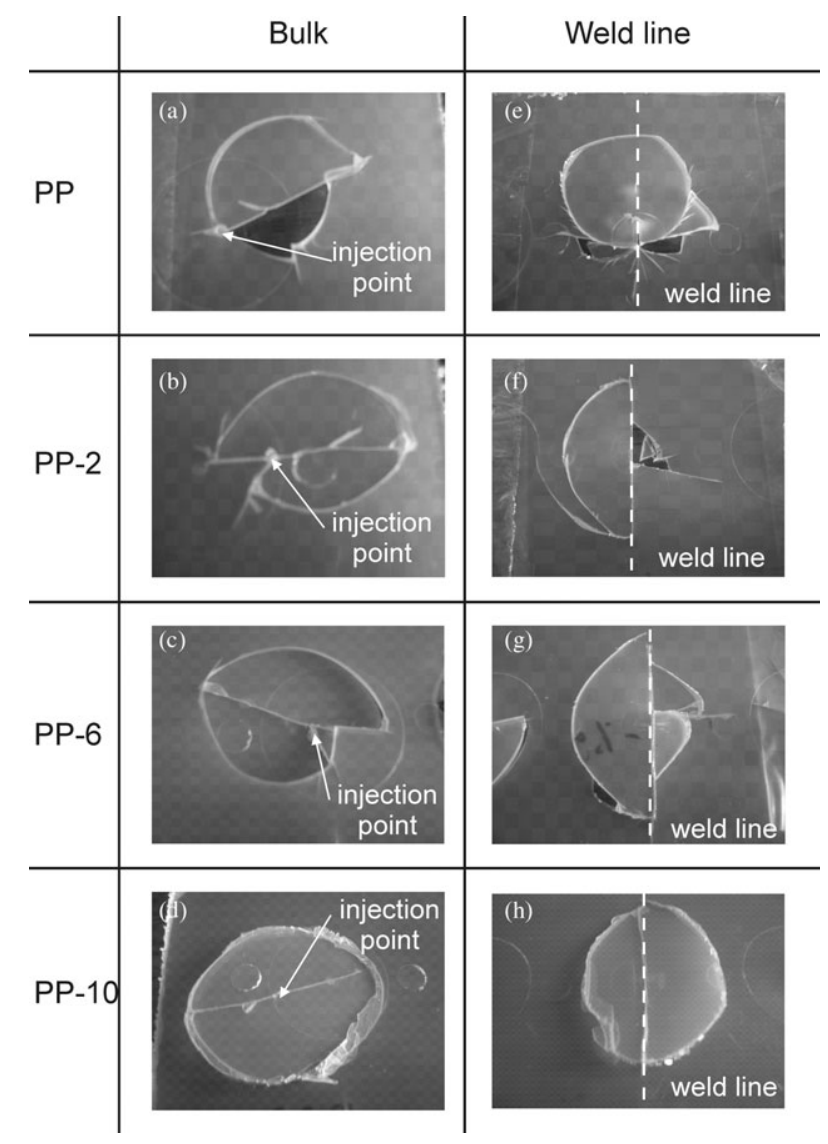

FIG. 12. Failure patterns of moldings at various locations: in the bulk for (a) PP (b) PP-2 (c) PP-6 (d) PP-10; and in the weld line for (e) PP (f) PP-2 (g) PP-6 (h) PP-10.

cantly different from that observed in the bulk. Assuming that the nanoclays are intercalated, this is due to the confinement of the polymer chain. An additional dissipative mechanism appears as a result of the mobility of the nanoparticles [35]. Since the nanofiller is interacting at the polymer chain level, the time scales for motion of both filler and polymer are comparable. As a result, during the deformation process the filler can create temporary polymer chain crosslinks, thereby creating a local region of enhanced strength and consequently retarding the growth of microdefects. However, since the mobility of the macromolecules is severely reduced when the temperature is below $\mathrm{T}_{\mathrm{g}}$, the ability of the nanofiller to contribute to the dissipation of energy is also reduced, resulting in almost no improvement in the toughness of the material. As PP is close to the glass transition at room temperature under impact conditions, it seems that this effect at the macromolecular scale can not be responsible for toughness improvement.

Cotterel et al. [36] showed that the delamination or splitting of nanoclay particles is another potentially significant source of toughness, this being related to the orientation of the nanoclay intercalated agglomerates with respect to the loading direction. In semi-crystalline polymers, like PP, shear yielding and craze-like bands are the mechanisms by which the matrix absorbs energy upon deformation. Nanosized free surfaces are necessary to initiate crazes and the delamination of clay particles produces those surfaces. Crazes can be readily initiated at the pole of delaminated nanoclay particles but not at the equator. Therefore, the favorable orientation of clay particles during injection molding can induce multiple crazing in tensile loaded samples and subsequently acting as a toughening mechanism. This is further improved by the delamination of the nanoclay agglomerates. In the case of biaxial impact tests, in which in-plane stresses are developed, these toughening effects are negligible, and fracture propagates in the direction of nanoclay orientation.

In summary, it seems that a failure mechanism which involves nanoclay delamination and multiple matrix crazing explains the toughening of PP in the directions where the nanoparticle orientation with respect to loading is adequate.

Analyzing the impact data, the best impact performance was observed in moldings with $6 \%$ of MB (corresponding to $3 \%$ of nanoclay). It seems that with higher nanoclay contents, as in microcomposites, their intercalation is reduced and the tactoids negatively act as defects, concentrating stresses.

\section{CONCLUSIONS}

In this article the possibility of getting impact performant $\mathrm{PP} /$ nanoclay parts by direct injection molding was explored, considering the influence of flow features inside the mold impression and nanoclay incorporation. Main findings are summarized in what follows.

The as-molded microstructure analyses showed that the nanoclay particles are mostly intercalated, and also that their presence induces the orientation of $\alpha$-PP crystallites and the reduction of $\beta$-form spherulites. The flow induced molecular orientation is enhanced by the presence of nanoclay which also causes the reduction of $\beta$-form spherulites, a known toughner.

The impact performance of these moldings in comparison to plain PP is practically not affected by the processing melt temperature and flow rate. However an ANOVA analysis showed that the nanoclay incorporation is the most influencing parameter in toughness of these nanocomposites. The weld lines are detrimental to the impact performance due to the macromolecular and nanoparticle flow induced orientations: in uniaxial testing and at the weld line their orientation is perpendicular to the applied force, whereas away from here the molecules and clay platelets are oriented with that force.

Under biaxial conditions, the moldings are more ductile and are tougher close to the weld lines than away from them. At the weld line zone the weakness caused by the molecular orientation prevailed, the weld lines being not determinant for the nanocomposite molding toughness: the difference between failure energies at bulk and at the weld line zone is negligible. 
It was made clear that under biaxial stresses the nanoclay does not improve the impact performance of PP, as it is governed by the molecular and nanoparticle orientations. However it seems that a failure mechanism which involves nanoclay delamination and multiple matrix crazing explains the toughening of PP in the directions where the nanoparticle orientation with respect to loading is adequate.

\section{REFERENCES}

1. J. Karger-Kocsis, Compos. Sci. Technol., 48, 273 (1993).

2. S. Sinha Ray and M. Okamoto, Prog. Polym. Sci., 28, 1539 (2003).

3. M. Alexandre and P. Dubois, Mater. Sci. Eng. R., 28, 1 (2000).

4. C. Ding, D. Jia, H. He, B. Guo, and H. Hong, Polym. Test., 24, 94 (2005).

5. Y.-Q. Zhang, J.-H. Lee, J.M. Rhee, and K.Y. Rhee, Compos. Sci. Technol., 64, 1383 (2004).

6. L. Chen, S.-C. Wong, T. Liu, X. Lu, and C. He, J. Polym. Sci. Polym. Phys., 42, 2759 (2004).

7. P.M. Frontini and A.S. Pouzada, Express Polym. Lett., 5, 661 (2011).

8. A.M. Cunha and A.S. Pouzada, "The Impact Behaviour of Polypropylene and the Injection-molding Conditions," in Impact and Dynamic Fracture of Polymers and Composites, G. Williams and A. Pavan, Eds., Mech. Eng. Publ., London, 315 (1995).

9. C.L. Morelli, J.A. de Sousa, and A.S. Pouzada, J. Vinyl Addit. Tech., 13, 159 (2007).

10. J.C. Viana, N. Billon, and A.M. Cunha, Polym. Eng. Sci., 44, 1522 (2004).

11. O.G. Ersoy and N. Nugay, Polymer, 45, 1243 (2004).

12. M.M. Gamba, A.S. Pouzada, and P.M. Frontini, Polym. Eng. Sci., 49, 1688 (2009).

13. S. Fellahi, A. Meddad, B. Fisa, and B.D. Favis, Adv. Polym. Tech., 14, 169 (1995).

14. A. Demirer, Y. Soydan, and A.O. Kapti, Mater. Des., 28, 1467 (2007).

15. P. Rosse, Taguchi Techniques for Quality Engineering, Loss Function, Orthogonal Experiments, Parameter and Tolerance Design, McGraw-Hill, Toronto (1996).
16. J.P. Trotignon, J.L. Lebrun, and J. Verdu, Plast. Rubber Proc. Appl., 2, 247 (1982).

17. P.-W. Zhu and G. Edward, Macromol. Mater. Eng., 288, 301 (2003).

18. W.C. Oliver and G.M. Pharr, J. Mater. Res., 7, 1564 (1992).

19. A.M. Cunha, A.S. Pouzada, and R.J. Crawford, Plast. Rubber Compos. Pro., 18, 79 (1992).

20. C.B. Bucknall, "Characterizing Toughness Using Standard Empirical Tests," in Polymer Blends, D.R. Paul and C.B. Bucknall, Eds., Wiley, New York, 25 (2000).

21. J.C. Viana, A.M. Cunha, and N. Billon, Polym. Int., 43, 159 (1997).

22. G. Kalay and M.J. Bevis, J. Polym. Sci. Polym. Phys. 35, 265 (1997).

23. J. Varga, J. Macromol. Sci. B, 41, 1121 (2002).

24. B. Kim, S.-H. Lee, D. Lee, B. Ha, J. Park, and K. Char, Ind. Eng. Chem. Res., 43, 6082 (2004).

25. M. Fujiyama, T. Wakino, and Y. Kawasaki, J. Appl. Polym. Sci., 35, 29 (1988).

26. M. Fujiyama, Int. Polym. Proc., 10, 172 (1995).

27. M. Fujiyama, Int. Polym. Proc., 10, 251 (1995).

28. M. Fujiyama, "Processing-induced Morphology," in Polypropylene: An AZ Reference, J. Karger- Kocsis, Ed., Kluwer Academic, Dordrecht, The Netherlands, 668 (1999).

29. L. Jiang, J. Zhang, and M.P. Wolcott, Polymer, 48, 7632 (2007).

30. G. Keppel and S. Zedeck, Data Analysis for Research Designs: Analysis of Variance and Multiple Research/ Correlation Approaches, W.H. Freeman, New York (1989).

31. A. Vaxman, M. Narkis, A. Siegmann, and S. Kenig, Polym. Compos., 12, 161 (1991).

32. A. Pozsgay, L. Papp, T. Fráter, and B. Pukánszky, "Polypropylene/Montmorillonite Nanocomposites Prepared by the Delamination of the Filler," in Adsorption and Nanostructure, I. Dékány, Ed., Springer, Berlin/Heidelberg, 120 (2002).

33. C.B. Bucknall, Pure Appl. Chem. 58, 985 (1986).

34. B. Alcock, N.O. Cabrera, N.M. Barkoula, and T. Peijs, Compos. Sci. Technol., 66, 1724 (2006).

35. D. Gersappe, Phys. Rev. Lett., 89, 058301 (2002).

36. B. Cotterell, J.Y.H. Chia, and K. Hbaieb, Eng. Fract. Mech., 74, 1054 (2007). 